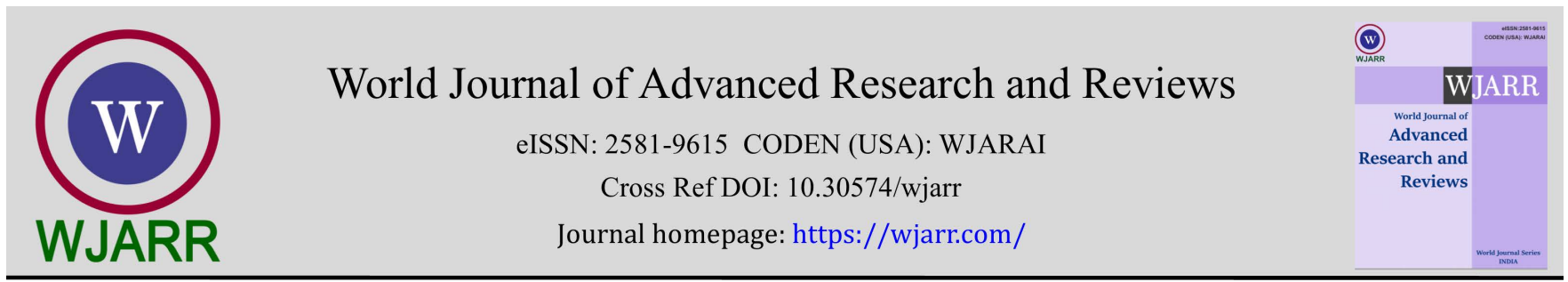

(RESEARCH ARTiCle)

Check for updates

\title{
Relationship between 6-minute walk test and 1-minute sit to stand test in patients with COVID-19: A retrospective analysis
}

\author{
Safa Liyaqatali Honpode $1^{*}$, Reema Shankar Rajam 1, Bhavana Suhas Mhatre 2, Snehal Sunil Sawant 1 \\ Vibhawari Manoj Wagh ${ }^{1}$ and Saraswati Iyer ${ }^{3}$ \\ ${ }_{1}$ Post-Graduate Student, Physiotherapy School \& Centre, Seth. G.S. Medical College \& KEM Hospital, Parel, Mumbai-12, \\ India. \\ 2 Professor, Physiotherapy School \& Centre, Seth. G.S. Medical College \& KEM Hospital, Parel, Mumbai-12, India. \\ ${ }^{3}$ Professor and Head, Physiotherapy School \& Centre, Seth. G.S. Medical College \& KEM Hospital, Parel, Mumbai-12, India.
}

World Journal of Advanced Research and Reviews, 2021, 12(02), 470-477

Publication history: Received on 15 October 2021; revised on 20 November 2021; accepted on 22 November 2021

Article DOI: https://doi.org/10.30574/wjarr.2021.12.2.0619

\begin{abstract}
Background: Coronavirus disease (COVID-19) is an infectious disease, causing a range of symptoms such as fever, cough, and dyspnea. 6 Minute walk test (6MWT) is recommended to measure functional capacity in COVID-19. 1- Min sit to stand $\left(\mathrm{STS}^{-1}\right)$ test has been used as its alternative in various respiratory conditions. This retrospective study aims to explore the relationship between the two tests in patients with COVID-19.
\end{abstract}

Patients and methods: The medical records of COVID-19 patients in a male step down unit, referred for physical therapy between the periods from 5th September, 2020 to 25th September, 2020 were analysed retrospectively. Hemodynamically stable patients who underwent 6MWT and STS ${ }^{-1}$ prior to discharge, were included in the analysis. SPSS Version 24 was used to find the correlation between 6-min walk distance (6MWD) and number of repetitions in $\mathrm{STS}^{-1}$; and to compare the haemodynamic responses between the two tests.

Results: There was a statistically significant positive correlation between the 6MWD and the STS ${ }^{-1}$ repetitions $(r=0.75$, $\mathrm{p}<0.0001)$. On comparison of the two tests, the change in heart rate (HR) was significantly greater in the $\mathrm{STS}^{-1}(p=$ 0.027). Whereas, the change in dyspnea (modified Borg's score) and $\mathrm{SpO}_{2}$ was similar in both the groups and was not statistically significant ( $\mathrm{p}=0.10, \mathrm{p}=0.62$; respectively).

Conclusion: There is a significant correlation between the 6MWT and STS ${ }^{-1}$ test with similar haemodynamic response and can perhaps be used as an alternative to 6MWT in COVID-19.

Keywords: COVID-19; 6-Minute Walk Test; 1- Minute sit to stand; Correlation

\section{Introduction}

Around December 2019, SARS-CoV-2, a new strain of coronavirus closely related to the one that causes SARS, was discovered in Wuhan, China [1]. This strain has caused the ongoing pandemic of COVID-19. Since its outbreak, $>27,000,000$ cases of COVID-19 have been accounted for globally as of now.

Majority of patients (81\%) report symptoms of mild disease, with fever, cough \& dyspnea being the most commonly reported. About 14\% develop severe illness, requiring oxygen therapy. Only 5\% develop critical illness with respiratory failure \& multiple organ dysfunction requiring intensive care unit (ICU) treatment [2].

\footnotetext{
* Corresponding author: Safa Liyaqatali Honpode

Post-Graduate Student, Physiotherapy School \& Centre, Seth. G.S. Medical College \& KEM Hospital, Parel, Mumbai-12, India.

Copyright (C) 2021 Author(s) retain the copyright of this article. This article is published under the terms of the Creative Commons Attribution Liscense 4.0.
} 
Patients with COVID-19 may have a rather normal ventilation but significant problems with oxygen diffusion because the underlying pathology is more of an inflammatory pneumonitis than a true pneumonia. Reduced oxygen diffusion is the cause of breathlessness and exercise intolerance, which impacts their physical activities of daily living [3]. Stefano Belli (2020) [4] has reported that during hospitalisation, 74.4\% patients had a number of sit to stand repetitions in 1 minute which was below percentile 2.5 of the reference value. Even after discharge, $33.3 \%$ of the patients were still below the percentile 2.5 of the reference values. He further concluded that COVID-19 patients who survived hospitalisation had low physical functioning and an impaired performance of ADLs [4].

The 6-minute walk test (6MWT) is a common test for assessing exercise tolerance (distance walked) and alterations in gas exchange (lowest oxygen saturation- $\mathrm{SpO}_{2}$ nadir) in most chronic lung diseases [5]. Even though it is sensitive, reproducible \& easy to perform, it does require a $30-\mathrm{m}$ corridor which is not always available in health centres and clinic practices. Shorter corridors require more turns, which is likely to distort the test results and the total distance travelled [6].

In recent years, to overcome these technical and spatial limitations, sit to stand (STS) tests like 1-min STS, 30-sec STS \& 5 times STS tests have been investigated. Studies have shown that 1-min STS is well tolerated, reproducible and sensitive in chronic obstructive pulmonary disease (COPD), interstitial lung disease (ILD] \& cystic fibrosis (CF] patients [7-9]. It requires only a chair \& is easy to perform even in confined spaces. Briand et al. [8] in a population with chronic interstitial lung disease, reported that the nadir $\mathrm{SpO}_{2}$ measured by oximetry on the 6MWT and the 1-min STS test showed high correlation ( $\mathrm{r}=0.9 ; \mathrm{p}<0.0001)$ and the number of repetitions on 1-min STS and 6- min walk distance showed moderate correlation $(\mathrm{r}=0.5, \mathrm{p}<0.0001)$. With respect to the exercise intensity, 1-min STS test has a MET value of $4.5 \pm 0.7$ METs, which is almost equivalent to moderate intensity exercises such as brisk walking at $5.6 \mathrm{~km} / \mathrm{hr}(4.3$ METs) [8]. Therefore, it has been suggested in literature that 1-min STS test can be used as an alternative for measuring functional capacity and detecting exercise-induced oxygen desaturation in healthy and diseased population such as ILD, COPD and cystic fibrosis [6-9]. The activity of sit to stand has also been used as an exercise programme to improve functional performance in hospitalized older adults [10].

6MWT is recommended for use in COVID-19 patients for measuring functional capacity pre-discharge [11]. However, with current increase in the numbers of patients each day, time and spatial constraints in each ward, performing and monitoring a walk test lasting for 6 minutes for each and every patient can become unfeasible. In the present retrospective analysis, we aim to find out if there is a correlation between 6MWT and 1-min STS test in terms of exercise tolerance and hemodynamic responsiveness in patients diagnosed with COVID-19.

\section{Material and methods}

\subsection{Subjects}

The medical records of COVID-19 patients in a male step down unit, referred for physical therapy between the periods from $5^{\text {th }}$ September, 2020 to $25^{\text {th }}$ September, 2020 were reviewed retrospectively. This study received institutional ethics approval and a waiver of consent was granted. (EC/OA-172/2020).

\subsection{Inclusion criteria}

- Diagnosis of COVID-19 with RT-PCR

- Hemodynamically stable ( $\mathrm{HR}<120 \mathrm{bpm}$ and $\mathrm{SpO}_{2}>95 \%$ at rest)

- Absence of dyspnea at rest

- Time from onset of symptoms $\geq 8$ days.

\subsection{Exclusion criteria}

Presence of any unstable cardiac, neurological or musculoskeletal impairments that may limit 6MWT or 1-min STS test performance.

\subsection{Clinical Measures:}

\subsubsection{6-Minute Walk Test}

6MWT was performed according to the guidelines of the American Thoracic Society. The test was executed by a physiotherapist who walked behind the patient. Patients were instructed to walk as far as they could and the distance walked was registered after 6 mins. During the test, standardised encouraging phrases were given to patients each 
minute. Patients who used walking aids in daily life were allowed to use the devices during the 6MWT. Heart rate, oxygen saturation $\left(\mathrm{SpO}_{2}\right)$ using pulse oximeter and intensity of dyspnea using Modified Borg's scale (0-10) were recorded pre and post the test.

\subsubsection{1-Minute Sit-to-Stand test}

The 1STST was performed with a stool of standard height $(46 \mathrm{~cm})$ without armrests positioned against a wall. The patient was seated upright with knees and hips flexed at $90^{\circ}$, feet placed flat on the floor a hip-width apart, and arms crossed across the chest. Patients were asked to perform repetitions of standing upright and then sitting down in the same position at a self-paced speed (safe and comfortable) as many times as possible for 1 minute. They were instructed not to use their arms for support while rising or sitting. Patients were permitted to rest during the 1-min period. The number of repetitions was recorded. Heart rate $(\mathrm{HR})$, oxygen saturation $\left(\mathrm{SpO}_{2}\right)$ using pulse oximeter and intensity of dyspnea using Modified Borg's scale (0-10) were recorded pre and post the test.

\subsection{Statistical analysis}

All analyses were conducted using SPSS version 24. Normality was verified by asymmetry coefficient and graphic analysis. The relationship of $1 \mathrm{~min}$ STS repetitions and 6MWD was calculated by Pearsons's correlation coefficient. This coefficient was also used to evaluate the relationship between $1 \mathrm{~min}$ STS repetitions \& 6MWD and the age of subjects. The paired t-test was used to measure the differences in cardiorespiratory parameters $\left(\mathrm{HR} \mathrm{SpO}_{2}\right.$ and intensity of dyspnea).

\section{Results}

\subsection{Demographic characteristics}

The demographic details have been presented in Table 1.

Table 1 Patient's baseline characteristics, and results of the tests

\begin{tabular}{|c|c|c|c|c|}
\hline Variable & Mean/n & S.D & Minimum & Maximum \\
\hline Male gender & 23 & - & - & - \\
\hline Age (years) & 47.7 & 16.6 & 20 & 79 \\
\hline Body mass index $(\mathrm{BMI})\left(\mathrm{kg} / \mathrm{m}^{2}\right)$ & 23.5 & 4.2 & 17.5 & 34.2 \\
\hline 6-minute walking distance (6MWD) (meters) & 197.7 & 55.6 & 60 & 300 \\
\hline Number of repetitions during STS ${ }^{-1}$ & 18.48 & 6.9 & 5 & 28 \\
\hline
\end{tabular}

Data are given as mean \pm SD; $\mathrm{STS}^{-1}=1$-min sit-to-stand test; 6MWT= 6-Minute Walk Test; HR= Heart Rate; $\mathrm{M}=$ mean; S.D = standard deviation

\subsection{Relationship between exercise capacity of $\mathrm{STS}^{-1}$ and the $6 \mathrm{MWT}$.}

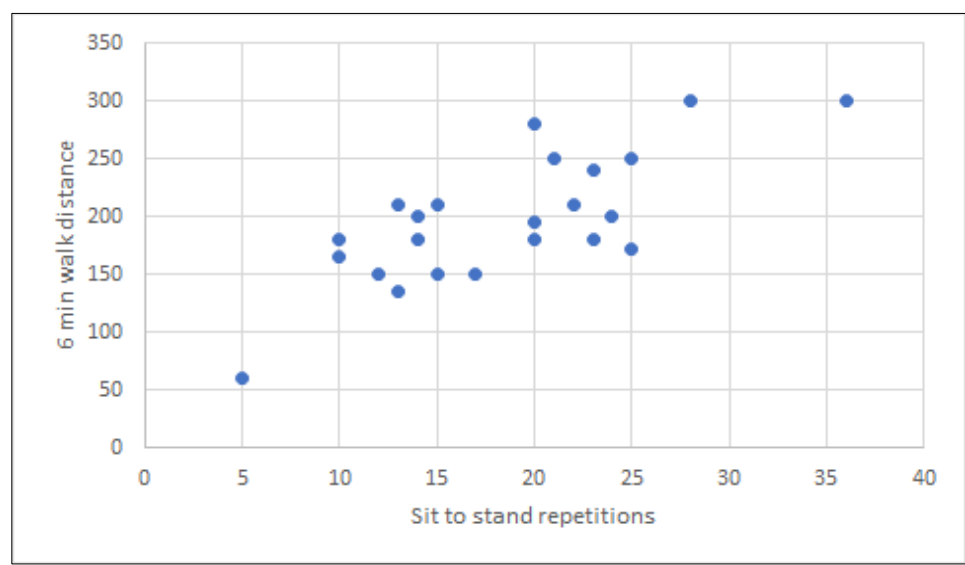

Figure 1 Relationship between 1-minute sit to stand repetitions and 6-min walk distance $(r=0.75, p<0.0001)$ 
There was a statistically significant positive correlation between the 6MWD and the STS $^{-1}$ repetitions $(r=0.75$, $\mathrm{p}<0.0001$ ). (Figure 1)

\subsection{Comparison of haemodynamic parameters between the $\mathrm{STS}^{-1}$ and the 6MWT}

The results of haemodynamic parameters and Modified Borg dyspnea, obtained during the STS ${ }^{-1}$ and 6MWT are presented in Table 2. The evolution for all the parameters are shown in Figures 2 to 4.

Table 2 Paired T-test for comparison of haemodynamic parameters between the STS ${ }^{-1}$ and the 6MWT

\begin{tabular}{|l|l|l|l|l|c|}
\hline Variables & Test & Baseline & End & Difference & P-value \\
\hline \multirow{2}{*}{ HR $(\mathrm{b} / \mathrm{min})$} & $6 \mathrm{MWT}$ & $94.39 \pm 18.8$ & $111.95 \pm 21.6$ & $17.56 \pm 18.3$ & \multirow{2}{*}{$0.027^{*}$} \\
\cline { 1 - 5 } & $\mathrm{STS}^{-1}$ & $94.3 \pm 17.1$ & $119.5 \pm 21.3$ & $25.17 \pm 15$ & \\
\hline \multirow{2}{*}{$\begin{array}{l}\text { Oxygen Saturation } \\
(\%)\end{array}$} & $6 \mathrm{MWT}$ & $97.5 \pm 1.9$ & $95.3 \pm 2.97$ & $2.21 \pm 2.35$ & \multirow{2}{*}{0.62} \\
\cline { 1 - 5 } & $\mathrm{STS}^{-1}$ & $97.3 \pm 1.98$ & $94.9 \pm 5.59$ & $2.39 \pm 2.49$ & \\
\hline \multirow{2}{*}{ Modified Borg's scale } & $6 \mathrm{MWT}$ & $0 \pm 0$ & $1.32 \pm 0.98$ & $1.32 \pm 0.98$ & \multirow{2}{*}{0.10} \\
\cline { 2 - 5 } & $\mathrm{STS}^{-1}$ & $0.06 \pm 0.22$ & $1.76 \pm 1.33$ & $1.69 \pm 1.21$ & \\
\hline
\end{tabular}

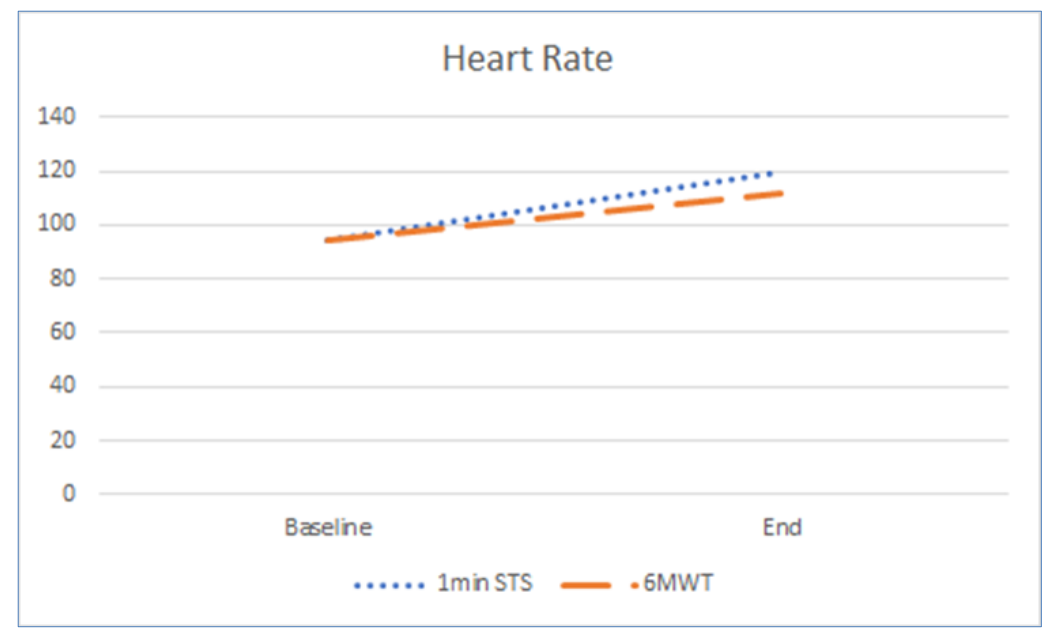

Figure 2 Evolution of heart rate in 1-min STS and 6MWT

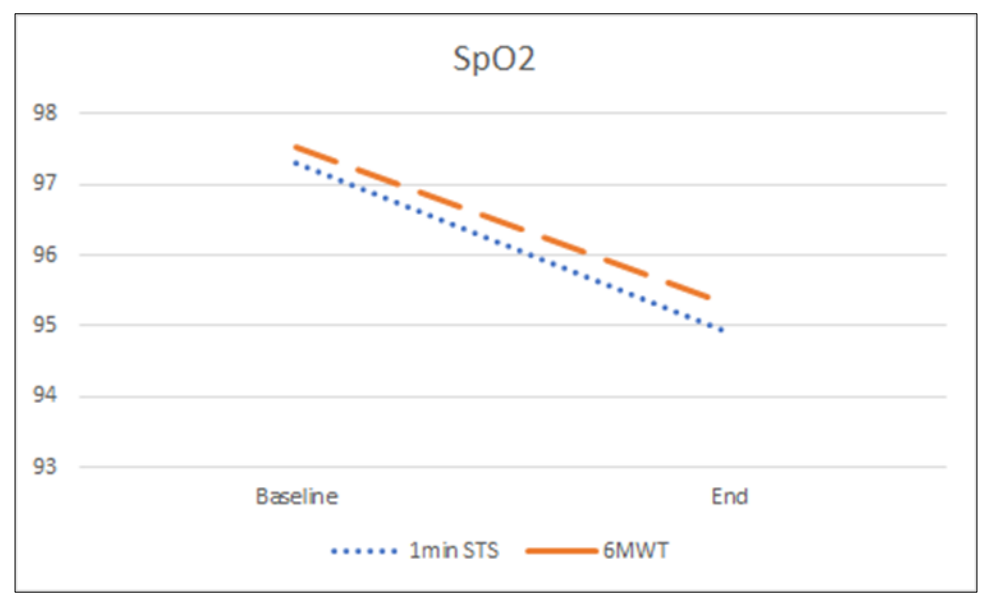

Figure 3 Evolution of oxygen saturation $\left(\mathrm{SpO}_{2}\right)$ in 1-min STS and 6MWT 


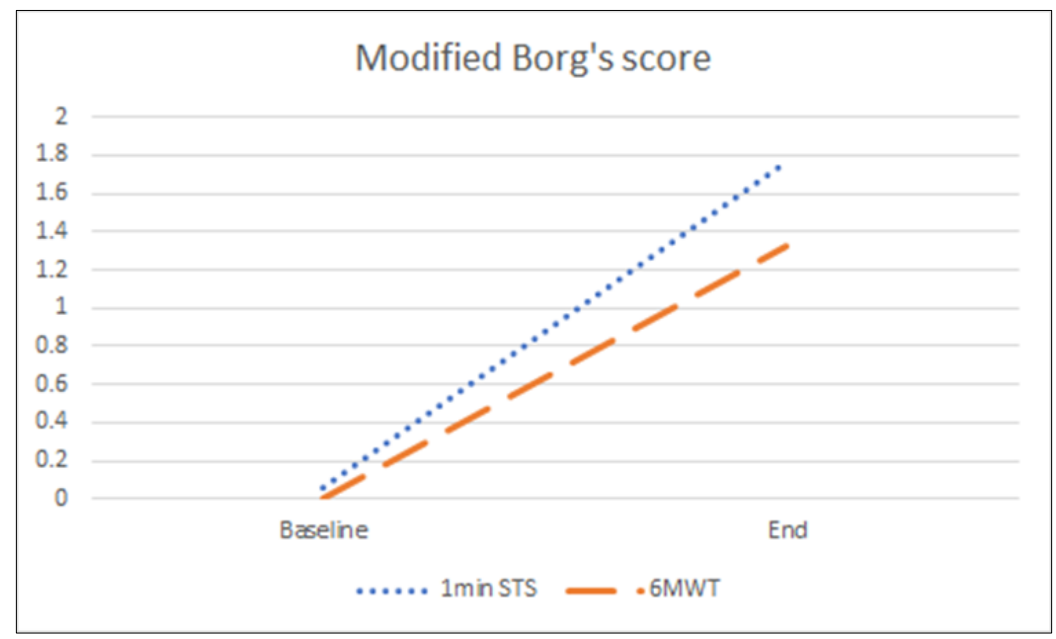

Figure 4 Evolution of dyspnea on Modified Borg's scale in 1-min STS and 6MWT

\subsection{Relationship of age with $1 \mathrm{~min} S T S$ reps and 6MWD}

Correlation analysis between the 1-minute sit-to-stand repetitions and Age are shown in Figure 5. STS ${ }^{-1}$ repetitions moderately associated with age. Increase in age was associated with reduction in number of sit-to-stand repetitions per minute $(r=-0.49, \mathrm{p}=0.0170)$.

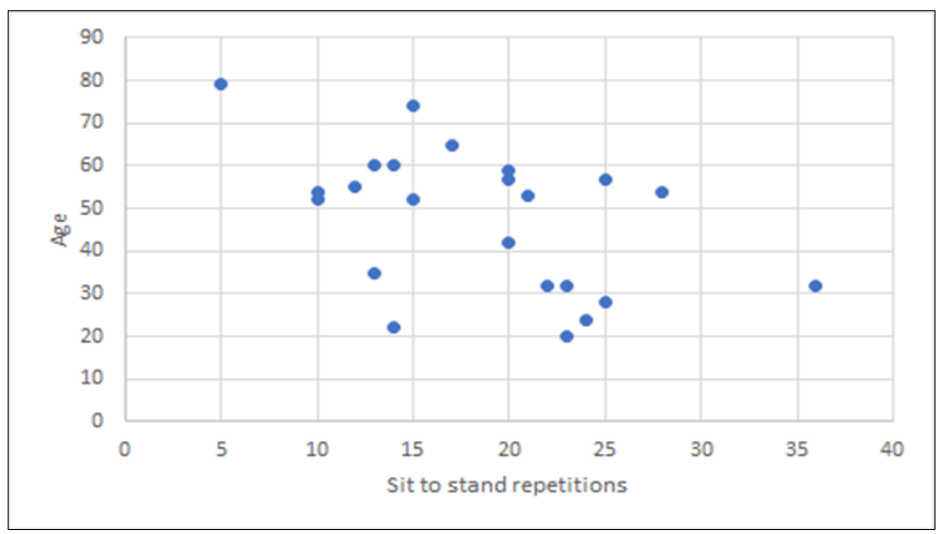

Figure 5 Relationship between 1-minute sit to stand repetitions and age

Correlation analysis between the 6-Minute Walk Test Distance and Age are shown below in Figure 6. The 6-Minute Walk Test Distance and Age did not shown any significant association $(r=-0.37, p=0.0745)$.

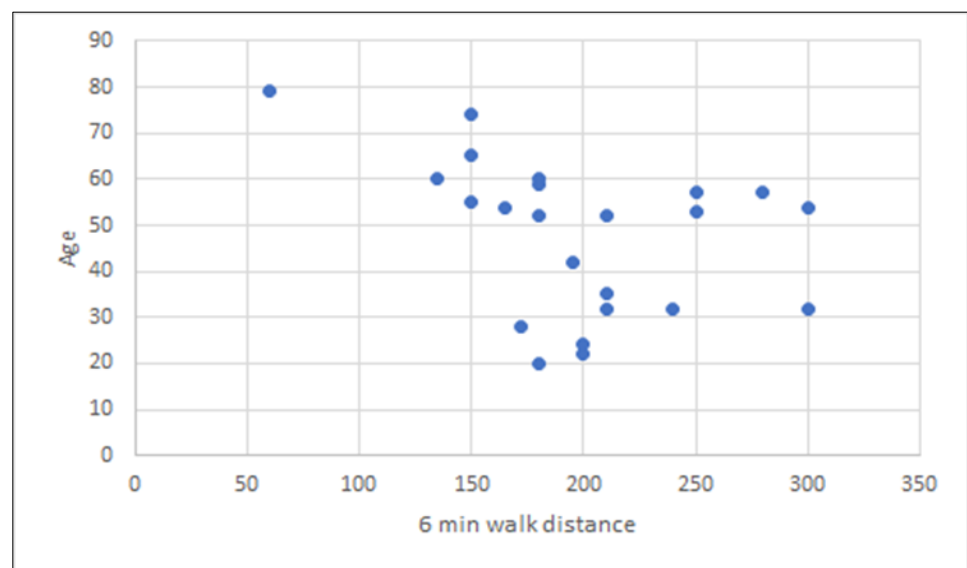

Figure 6 Relationship between 6-min walk distance and age 


\section{Discussion}

To the best of our knowledge and literature search, we did not find any study that has retrospectively analysed the relationship between 6MWT and 1 min STS test in SARS COVID-19. However, similar studies have previously established a moderate to strong positive correlation between 6MWT Distance and 1min STS repetitions in patients with cystic fibrosis, ILD and COPD. The results of this present retrospective analysis were in agreement with these findings and found a strong positive correlation between 1min STS repetitions and 6MWD in patients with COVID-19 $(\mathrm{r}=0.75, \mathrm{p}<0.0001)$. (Figure 1)

Measuring functional capacity in COVID-19 becomes particularly important as a recent study demonstrated low physical functioning and impaired performance of ADLs even after discharge in patients who survived hospitalization due to COVID-19 [4].

Reduction in functional capacity can have deleterious effects on patient's independence, increased health care costs and reduced quality of life [QOL), especially in older adults. Early identification and addressal of these deficits can probably intercept these issues.

The 6MWT, is a commonly used outcome measure for functional capacity in healthy and diseased populations such as ILD, COPD and CF [7-9]. Upcoming literature suggests its use in COVID-19 patients, for not only discharge preparedness but also in early detection of hypoxia for initiating early higher-level care [12][13]. Due to the rising number of COVID 19 cases, constraints such as a monitored walk for 6 minutes and a 30 meter corridor can make it infeasible to perform in health-care setups. Whereas the 1 minute sit-to-stand test is less time consuming that requires only a standard height chair and minimal space. It can also be used to test individuals with severely limited mobility. The results of this present retrospective analysis found a strong positive correlation between 1min STS repetitions and 6MWD ( $\mathrm{r}=0.75$, $\mathrm{p}<0.0001)$. Also, since both the tests involve movements used in activities of our daily living and are used to measure functional capacity in healthy \& diseased conditions, it can be suggested to use $1 \mathrm{~min}$ sit-to-stand test as an alternative to $6 \mathrm{MWT}$.

However, in contrast to the studies on COPD and CF our study indicated increased heart rate in $1 \mathrm{~min}$ sit to stand test compared to 6MWT [STS-1 $\Delta \mathrm{HR}: 26.7 \%, 6 \mathrm{MWT} \Delta \mathrm{HR}: 18.5 \%$; $(\mathrm{p}=0.027)$ ] as shown in Table 2. On literature appraisal, we found greater distance covered associated with a higher change in heart rate in 6MWT by patients with COPD, ILD and cystic fibrosis [7-9]. However, the change in HR for 6MWT was less, likely due to the shorter 6MWD covered by patients with COVID-19 in our study. The only patient reported measure in our study was dyspnea on modified Borg's scale and not rate of perceived exertion (RPE) by Borg's scale for fatigue. It is possible that fatigue played a role in the shorter 6MWD covered and increased change in HR for the sit to stand activity due to the greater muscle work required.

The activity of STS requires greater quadriceps and gluteal strength. EMGs have shown that the quadriceps, biceps femoris, gluteus maximus, and tibialis anterior are active during all the phases of sit-to-stand activity, producing a greater joint torque at the knee, followed by the hip and ankle [14]. Hence, it could be said that the activity of sit to stand was more physically demanding compared to level surface walking [6]. It is also dependent on other factors associated with balance and mobility at hip, knee and ankle. Recent literature also suggests possible musculoskel etal changes such as elevated CK levels and lactate dehydrogenase which can contribute to muscle damage and increased muscle fatigue in patients with COVID-19 [15]. Another possible contributor could be the exercise pressor reflex, which increases the heart rate to increase the cardiac output and is directly proportional to the intensity of the exercise [16]. However, the change in saturation was not statistically different between the tests [STS ${ }^{-1} \Delta \mathrm{SpO}_{2}: 2.4 \%, 6 \mathrm{MWT} \Delta \mathrm{SpO}_{2}: 2.2 \%$; $\left.(\mathrm{p}=0.62)\right]$. This was in accordance with studies on oxygen desaturation in interstitial lung disease, cystic fibrosis and COPD. $[8,9][17,18]$.

There was no significant correlation between age and 6MWD ( $\mathrm{r}=-0.37, \mathrm{p}=0.0745)$. However, analysis demonstrated a strong negative correlation between STS repetitions and age, suggesting reduction in functional capacity with increase in age $(r=-0.49, \mathrm{p}=0.0170)$ (Table 3$)$. Frequent and regular functional assessments, especially in older adults can aid in early detection of functional decline which is important to start interventions at an early state and to prolong the functional capacity.

As physical therapists, we play a vital role in acute care settings as well as in post COVID rehabilitation, with interventions ranging from positioning, mobilisation to improving the functional capacity of patients. Studies have demonstrated 1-minute sit-to-stand test as a good tool for the evaluation of the impact of pulmonary rehabilitation and established 3 repetitions as the minimally clinically important difference (MCID) for COPD. The sit to stand activity apart from an evaluation tool can also be utilised for therapy to improve balance, mobility and strength. It is feasible to 
perform, progress and regress with no adverse events in a hospital as well as a home setting.[10,19]. This provides a future scope for studies utilising 1min sit-to-stand tests in COVID-19.

\section{Abbreviations}

COVID-19: Coronavirus disease 19

6MWT: 6- Minute Walk Test

6MWD: 6- Minute Walk Distance

STS $^{-1}: 1$ - Minute Sit to Stand

COPD: Chronic Obstructive Pulmonary Disease

ILD: Interstitial Lung Disease

CF: Cystic Fibrosis

HR: Heart Rate

$\mathrm{SpO}_{2}$ : Oxygen saturation

RT-PCR: Reverse transcription polymerase chain reaction

MET: Metabolic Equivalent

QOL: Quality of Life

MCID: Minimal Clinical Important Difference

\section{Conclusion}

There is a significant correlation between the 6MWT and $\mathrm{STS}^{-1}$ test with similar haemodynamic response and can perhaps be used as an alternative to 6MWT in COVID-19.

\section{Compliance with ethical standards}

\section{Acknowledgments}

The authors acknowledge Dr. Hemant Deshmukh (Dean, Seth GSMC \& KEM Hospital Mumbai), Dr. Milind Nadkar (Academic Dean, Seth GSMC \& KEM Hospital), Dr. Mariya P Jiandani (Associate Professor, Physiotherapy school and center, Seth GSMC and KEMH), Dr. Rupali Deshpande (Assistant physiotherapist, Physiotherapy school and center, Seth GSMC and KEMH), and the patients whose information was used.

\section{Disclosure of conflict of interest}

No conflict of interest.

\section{Statement of informed consent}

Not Applicable, retrospective study.

\section{References}

[1] Li Q, Guan X, Wu P, et al.Early Transmission Dynamics in Wuhan, China, of Novel Coronavirus-Infected Pneumonia. N Engl J Med. 26 Mar 2020; 382(13): 1199-1207.

[2] Chang D, Mo G, Yuan X, et al. Time Kinetics of Viral Clearance and Resolution of Symptoms in Novel Coronavirus Infection. Am J Respir Crit Care Med. 1 May 2020; 201(9): 1150-1152.

[3] Dondorp AM, Hayat M, Aryal D, et al.Respiratory Support in COVID-19 Patients, with a Focus on Resource-Limited Settings. Am J Trop Med Hyg. Jun 2020; 102(6): 1191-1197.

[4] Belli S, Balbi B, Prince I, et al. Low physical functioning and impaired performance of activities of daily life in COVID-19 patients who survived hospitalisation. Eur Respir J. 15 Oct 2020; 56(4): 2002096.

[5] ATS Committee on Proficiency Standards for Clinical Pulmonary Function Laboratories. ATS statement: guidelines for the six-minute walk test. Am J Respir Crit Care Med. 1 Jul 2002; 166(1): 111-7.

[6] Gurses HN, Zeren M, Denizoglu Kulli H, et al. The relationship of sit-to-stand tests with 6-minute walk test in healthy young adults. Medicine (Baltimore). Jan 2018; 97(1): e9489. 
[7] Reychler G, Boucard E, Peran L, et al. One minute sit-to-stand test is an alternative to 6MWT to measure functional exercise performance in COPD patients. Clin Respir J. Mar 2018; 12(3): 1247-1256.

[8] Briand J, Behal H, Chenivesse C, et al.The 1-minute sit-to-stand test to detect exercise-induced oxygen desaturation in patients with interstitial lung disease. Ther Adv Respir Dis. Jan-Dec 2018; 12: 1753466618793028.

[9] Gruet M, Peyré-Tartaruga LA, Mely L, et al. The 1-Minute Sit-to-Stand Test in Adults with Cystic Fibrosis: Correlations with Cardiopulmonary Exercise Test, 6-Minute Walk Test, and Quadriceps Strength. Respir Care. Dec 2016; 61(12): 1620-1628.

[10] Pedersen MM, Petersen J, Bean JF, et al.Feasibility of progressive sit-to-stand training among older hospitalized patients. PeerJ. 17 Dec 2015; 3: e1500.

[11] Jiandani MP, Agarwal B, Baxi G, et al. Evidence-based National Consensus: Recommendations for Physiotherapy Management in COVID-19 in Acute Care Indian Setup. Indian J Crit Care Med. Oct 2020; 24(10): 905-913.

[12] Pandit R, Vaity C, Mulakavalupil B, et al. Unmasking Hypoxia in COVID 19 - Six Minute Walk Test. J Assoc Physicians India. Sep 2020; 68(9): 50-51.

[13] Mantha S, Tripuraneni SL, Roizen MF, et al. Proposed Modifications in the 6-Minute Walk Test for Potential Application in Patients with Mild COVID-19: A Step to Optimize Triage Guidelines. Anesth Analg. Aug 2020; 131(2): 398-402.

[14] Mak MK, Levin O, Mizrahi J, et al. Joint torques during sit-to-stand in healthy subjects and people with Parkinson's disease. Clin Biomech (Bristol, Avon). Mar 2003; 18(3): 197-206.

[15] Mao L, Jin H, Wang M, et al. Neurologic Manifestations of Hospitalized Patients With Coronavirus Disease 2019 in Wuhan, China. JAMA Neurol. 1 Jun 2020; 77(6): 683-690.

[16] Gallagher KM, Fadel PJ, Strømstad M,et al. Effects of exercise pressor reflex activation on carotid baroreflex function during exercise in humans. J Physiol. 15 Jun 2001; 533(3): 871-80.

[17] Fernandes AL, Neves I, Luís G, et al. Is the 1-Minute Sit-To-Stand Test a Good Tool to Evaluate Exertional Oxygen Desaturation in Chronic Obstructive Pulmonary Disease? Diagnostics (Basel). 22 Jan 2021; 11(2): 159.

[18] Vaidya T, de Bisschop C, Beaumont M, et al. Is the 1-minute sit-to-stand test a good tool for the evaluation of the impact of pulmonary rehabilitation? Determination of the minimal important difference in COPD. Int J Chron Obstruct Pulmon Dis. 19 Oct 2016; 11: 2609-2616.

[19] Rosie J, Taylor D. Sit-to-stand as home exercise for mobility-limited adults over 80 years of age--GrandStand System may keep you standing? Age Ageing. Sep 2007; 36(5): 555-62. 